\section{Enfermera del ámbito de la geriatría de Barcelona. Situación socioeconómica, laboral y percepción de su rol}

\section{Nurses in geriatric field of Barcelona. Socioeconomic situation, labour and role perception}

\author{
Ester López Luna ${ }^{1, *}$ \\ Miquel Perea García \\ Noemí Sech Macías ${ }^{3}$ \\ Imma Mundet Riera ${ }^{4}$ \\ Elvira Hernández Martínez-Esparza ${ }^{5}$ \\ Ainhoa Torner Busquets ${ }^{6}$ \\ Núria Mateu Olivé ${ }^{7}$ \\ Mireia Filip Ayala ${ }^{8}$
}

\author{
1. Enfermera. Especialista en geriatría. HSS Mutuam Güell. Barcelona. España. \\ 2. Enfermero. Especialista en geriatría. Hospital de Sant Celoni. Barcelona. España. \\ 3. Enfermera. Especialista en geriatría. CR Mutuam Collserola. Barcelona. España. \\ 4. Enfermera. Especialista en geriatría. EAR Mutuam. Barcelona. España. \\ 5. Enfermera. Especialista en geriatría. EUI-Sant Pau. Barcelona. España. \\ 6. Enfermera. Especialista en geriatría. Responsable de Calidad. Grup Mutuam. Barcelona. España. \\ 7. Enfermera. Máster en geriatría. Hospital de Granollers. Barcelona. España. \\ 8. Enfermera. Especialista en geriatría. HSS Mutuam Güell. Barcelona. España. \\ ${ }^{*}$ Autor para correspondencia. \\ Correo electrónico: esterlop73@gmail.com (Ester López Luna).
}

Recibido el 6 de septiembre de 2019; aceptado el 9 de diciembre de 2019.

\section{RESUMEN}

Objetivo: Determinar y describir la situación laboral de las enfermeras del ámbito de la geriatría de la provincia de Barcelona, así como su percepción.

Metodología: Estudio descriptivo transversal, a través de una encuesta online anónima enviada a las enfermeras registradas o que habían realizado formación en geriatría en el COIB (Col-legi Oficial d'Infermeres i Infermers de Barcelona). La encuesta ad hoc consistía en 40 preguntas, mayoritariamente con respuestas múltiples cerradas, sobre datos relativos al lugar de trabajo y percepción. Resultados: El 91,4\% eran mujeres, el 50,5\% con más de 15 años de experiencia y el 59\% tenía amplia experiencia en geriatría. Más de la mitad tienen formación específica, el 67,4\% posgrado y el $41 \%$ máster. El 80,5\% trabajaba exclusivamente en el ámbito asistencial, ubicadas en mayor proporción en centros sociosanitarios (36,5\%) y residencias (40\%). Tres de cada cuatro enfermeras tenían jornada completa y explicaban tener a su cargo más de 50 pacientes el 36,5\% de ellas. El lugar de trabajo con sueldo más alto fue en los centros sociosanitarios, y el $69 \%$ no consideraba su sueldo adecuado. El $73,5 \%$ indicaba realizar siempre tareas asistenciales, pero el $58 \%$ nunca realizaba tareas de investigación o docencia. El 68,5\% eligió la geriatría por decisión propia y el 69\% no sentía reconocida su profesión. Conclusiones: Aunque las condiciones laborales en el ámbito de la geriatría constan de sueldos bajos, alto ratio de pacientes, poco tiempo para la investigación y percepción de poco reconocimiento, las enfermeras del ámbito de la geriatría de Barcelona sienten satisfacción profesional por su trabajo.

PALABRAS CLAVE: Enfermera geriátrica, condiciones laborales, ratios, tareas, satisfacción.

\section{ABSTRACT}

Objective: Define, describe and discover the perception about the work situation of nurses in the geriatric field of Barcelona province. Methods: Descriptive cross-sectional study based in an anonymous survey sent to all the registered nurses or those who have been training in geriatrics in the COIB. The survey ad hoc consisted on 40 questions, most closed multiple choice related to workplace and perception. Results: $91.4 \%$ were women, $50.5 \%$ with more than 15 years of experience and the $59 \%$ had extensive experience in geriatrics. More than a half of the nurses had specific training, $67.4 \%$ a postgraduate degree and $41 \%$ a master's degree. $80.5 \%$ worked exclusively in health care area, they were in larger proportion in long-term care facility $36.5 \%$ and $40 \%$ in a nursing home. Three out of four nurses had full-time work and $36.5 \%$ explained that they had over fifty patients. The workplace with a higher salary was longterm care facilities and $69 \%$ of the nurses didn't think their salary is adequate. $73.5 \%$ always performed care tasks but $58 \%$ of them never had done teaching or research tasks. $68.5 \%$ explained that work in geriatrics was their own decision and 69\% felt that people didn't value their work. Conclusions: Although the labour conditions in geriatric field have low salaries, high ratio of patients, very little time to research and the feeling of people not valuing their work; the geriatric nurses of Barcelona feel job satisfaction.

KEYWORDS: Geriatric nurse, labour conditions, ratios, tasks, satisfaction.

\section{E INTRODUCCIÓN}

Desde 2013, con la especialidad de enfermería geriátrica ${ }^{1}$ por la vía excepcional, se afianzó la figura de las enfermeras en geriatría ${ }^{2,3}$. Esto, junto a la demanda asistencial creciente dado el envejeci- miento poblacional ${ }^{4}$ y la prevalencia de patologías crónicas ${ }^{5}$, ha hecho que el papel de la enfermera que trabaja en el ámbito de la geriatría haya adquirido importancia en los últimos ańos. Incluso se han creado nuevos recursos específicos de geriatría, como, por ejemplo, equipos de atención residencial (EAR) ${ }^{(1)}$, unidad de valo- 
ración geriátrica integral $(\mathrm{UVGI})^{(2)}$, dispositivo de atención a la fragilidad del servicio de urgencias (DAFSU) ${ }^{(3)}$ y equipo de soporte integral a la complejidad (ESIC) ${ }^{(4)}$, entre otros. Estos recursos no siempre están dotados de enfermeras especialistas en geriatría, aunque la enfermera especialista en geriatría desempeńa un papel importantísimo en diferentes ámbitos asistenciales, dada su experiencia y formación ${ }^{6}$.

Pero, a pesar de ser tan importante a nivel asistencial, parece que las condiciones laborales no han evolucionado en consonancia al rol de la especialista en geriatría y se desconoce su actividad real laboral, los ratios enfermera-paciente, funciones, tareas, así como su percepción del rol y satisfacción del mismo ${ }^{7}$.

\section{- OBJETIVOS}

Objetivo general: Describir la situación laboral de las enfermeras del ámbito de la geriatría de la provincia de Barcelona, así como su percepción.

Objetivos específicos:

- Determinar la actividad real realizada por las enfermeras que trabajan en el ámbito de la geriatría de la provincia de Barcelona.

- Describir los datos relativos a la percepción de su situación laboral, respecto a ratios de pacientes, tipos de contrato y remuneración, funciones y tareas desarrolladas en la jornada laboral.

- Determinar la percepción de las enfermeras del ámbito de la geriatría de la provincia de Barcelona sobre su imagen profesional y grado de satisfacción.

\section{E METODOLOGÍA}

Estudio descriptivo transversal, a través de una encuesta online, anónima. La población diana de estudio fueron las enfermeras del ámbito de la geriatría de la provincia de Barcelona. Para ello, se envió la encuesta a todas las enfermeras registradas en la base de datos del Col.legi Oficial d'Infermeres i Infermers de Barcelona (COIB) que habían registrado la especialidad de geriatría $(\mathrm{n}=287)$ o que habían realizado alguna formación relacionada con la geriatría en el COIB, o que en la base de datos constase que estaban trabajando en el ámbito de la geriatría. La encuesta ad hoc consistía en 40 preguntas, en su mayoría con respuestas múltiples cerradas, sobre datos relativos al lugar de trabajo y percepción. Se realizó previamente una prueba piloto entre enfermeras especialistas en geriatría para constatar el entendimiento y la correcta recogida de información, y no hubo que realizar ningún cambio sobre la encuesta inicial. Los datos se recogieron desde noviembre de

(1) Dispositivo sanitario asistencial formado por médicos y enfermeras expertas en geriatría que coordinados con EAP y los profesionales de residencias mejoran la atención a las personas frágiles y en régimen de internamiento residencial.

(2) Recurso de la red sanitaria pública que da soporte especializado a pacientes geriátricos de atención primaria de Barcelona. Formados por expertos en geriatría, enfermera neuropsicóloga y trabajadora social.

(3) Nuevo dispositivo en el marco del programa de atención la fragilidad del servicio de urgencias y enmarcado dentro del plan nacional de urgencias de Cataluña (PLANUC).

(4) Equipo único de profesionales de atención primaria y hospitalaria que trabajan de manera colaborativa las visitas a urgencias para prevenir descompensaciones y complicaciones en los pacientes crónicos complejos.
2016 hasta febrero del 2017. Se realizó un envío a 649 individuos que en la base de datos constara que trabajaban o tenían relación con el ámbito y/o entorno de geriatría gerontología (disponer del título de especialista, haber hecho cursos de formación sobre el tema, haber registrado que trabajan en geriatría, etc.), lo que supone un 2,01\% de los colegiados con alta ejerciente (32 266 enfermeras). Para el análisis de resultados, las variables categóricas se representaron mediante frecuencia absoluta y porcentaje (\%). En todo momento se mantuvo la confidencialidad de los datos, según lo dispuesto en la Ley Orgánica 3/2018, de 5 de diciembre, de Protección de Datos Personales y garantía de los derechos digitales. La proporción de los datos por parte de la enfermera participante era anónima y suponía un consentimiento implícito de participación.

\section{- RESULTADOS}

Se obtuvieron un total de 200 encuestas, lo que supuso el 30,81\% del total de encuestas enviadas. No todas las encuestas contenían todas las respuestas.

\section{Características generales de las enfermeras del ámbito de la geriatría}

La gran mayoría de los encuestados eran mujeres $(91,4 \%)$, con una edad de entre 30 y 49 años (71,8\%). Respecto a los años que llevaban ejerciendo como enfermeras, la mitad $(50,5 \%)$ ejercía desde hace más de 15 años y el 10,5\% desde hace menos de 5 años. Si observamos la experiencia profesional en el ámbito de geriatría concretamente, más de la mitad (59\%) ejercía desde hace más de 11 años y el 15\% desde hace menos de 5 años. El 52,5\% tenía la especialidad de geriatría, y el 93,3\% de ellos la habían obtenido por la vía excepcional. Respecto a la formación específica en geriatría, de las 95 personas que responden, el 67,4\% tienen un posgrado y el $41 \%$ un máster de geriatría.

\section{Actividad laboral de las enfermeras del ámbito de la geriatría}

El 97,5\% (195) de las enfermeras estaban en activo en el momento de la encuesta, y de estas, 157 (80,5\%) trabajaban exclusivamente en el ámbito asistencial, 1 persona $(0,5 \%)$ en docencia, 12 (6,1\%) en gestión, 25 $(12,8 \%)$ trabajaban en varios ámbitos a la vez y ninguno exclusivamente en investigación.

Los recursos asistenciales donde trabajaban (fig. 1) fueron mayoritariamente las residencias (40\%) y los centros sociosanitarios (36,5\%). El resto $(23,5 \%)$ desarrollaba su labor asistencial en PADES (Programa de Atención a Domicilio y Equipos de Soporte), EAR (equipo de atención residencial), hospital de día, centros de día, UFISS (unidad funcional interdisciplinaria sociosanitaria) y otros. El 14,5\% trabajaba en más de un sitio, y de estos, el 43,2\% de estos segundos puestos de trabajo están relacionados con la geriatría. Respecto a la naturaleza de los centros empleadores, el $56 \%$ trabajaba en centros concertados, el $25 \%$ privados y el $15 \%$ públicos (omitidas un $4 \%$ ).

Respecto al tipo de jornada, los turnos de trabajo y los contratos, tres de cada cuatro enfermeras tenían jornada completa (el 76,5\%), el 20\% jornada parcial y un 3,5\% ha omitido la respuesta. Casi la mitad de las encuestadas (48\%) trabajaba en turno de mañana, un $13 \%$ en turno de tarde y el $7 \%$ en turno de noche. Un 28,5\% tenía un turno partido o rotatorio. La mayoría de las enfermeras tenían contrato indefinido (el $80,5 \%)$ (tabla 1). 
Tabla 1. Turno y contrato versus ratio de ancianos por enfermera

\begin{tabular}{|c|c|c|c|c|c|c|}
\hline & \multicolumn{6}{|c|}{$\mathrm{N}^{0}{ }^{0}$ de personas atendidas por enfermera } \\
\hline & Entre 10 y 30 & Entre 31 y 50 & Entre 51 y 100 & Más de 100 & En blanco & Total \\
\hline \multicolumn{7}{|c|}{ Turno de la enfermera } \\
\hline Mañana & $27(13,5)$ & $31(15,5)$ & $19(9,5)$ & $14(7)$ & $5(2,5)$ & $96(48)$ \\
\hline Tarde & $10(5)$ & $2(1)$ & $8(4)$ & $4(2)$ & $2(1)$ & $26(13)$ \\
\hline Noche & $4(2)$ & $3(1,5)$ & $4(2)$ & $3(1,5)$ & $1(0,5)$ & $15(7,5)$ \\
\hline Partido & $6(3)$ & $6(3)$ & $8(4)$ & $3(1,5)$ & $3(1,5)$ & $26(13)$ \\
\hline Rotatorio y otros & $10(5)$ & $4(2)$ & $8(4)$ & $2(1)$ & $5(2,5)$ & $29(14,5)$ \\
\hline \multirow[t]{2}{*}{ En blanco } & $0(0)$ & $0(0)$ & $0(0)$ & $0(0)$ & $8(4)$ & $8(4)$ \\
\hline & $57(28,5)$ & $46(23)$ & $47(23,5)$ & $26(13)$ & $24(12)$ & $200(100)$ \\
\hline \multicolumn{7}{|c|}{ Tipo de contrato de la enfermera } \\
\hline Indefinido & $50(25)$ & $43(21,5)$ & $38(19)$ & $19(9,5)$ & $11(5,5)$ & $161(80,5)$ \\
\hline Eventual & $7(3,5)$ & $3(1,5)$ & $9(4,5)$ & $7(3,5)$ & $5(2,5)$ & $31(15,5)$ \\
\hline \multirow[t]{2}{*}{ En blanco } & $0(0)$ & $0(0)$ & $0(0)$ & $0(0)$ & $8(4)$ & $8(4)$ \\
\hline & $57(28,5)$ & $46(23)$ & $47(23,5)$ & $26(13)$ & 24 (12) & $200(100)$ \\
\hline
\end{tabular}

Los datos se muestran en valores absolutos y porcentajes.

\section{Situación laboral de las enfermeras del ámbito de la geriatría}

Como se observa en la tabla 1, el 36,5\% de las enfermeras encuestadas referían atender a más de 50 pacientes en su turno, un $23 \%$ entre 31 y 49 y un $28,5 \%$ entre 10 y 30 . Las residencias geriátricas fueron el recurso donde mayor número de pacientes/residentes había por enfermera; en la mayoría, la media fue de 50 a 100 pacientes por enfermera. En los centros sociosanitarios, la mediana de pacientes fue de \pm 40 pacientes por enfermera, pero podían llegar hasta los 100 . Y en los centros de día, de hasta \pm 40 pacientes por enfermera (fig. 1).

Los lugares de trabajo con salarios más altos fueron los centros sociosanitarios, y los más bajos, las residencias geriátricas (fig. 2). El 69\% de los entrevistados no consideran adecuado su sueldo (omisión 6\%). El $32,5 \%$ desconocía el convenio que se aplicaba en su centro de trabajo.
- FUNCIONES Y TAREAS DESARROLLADAS EN LA JORNADA LABORAL POR LA ENFERMERA DEL ÁMBITO DE LA GERIATRÍA

En la figura 3 se muestran todos los datos respecto a las tareas según recurso asistencial. El 73,5\% de las enfermeras indicaron realizar "siempre" tareas asistenciales, el 51\% realizaba "a menudo" o "siempre" tareas de gestión, el 58\% "nunca" tareas de investigación y docencia, y el 44\% "ocasionalmente" tareas de docencia/formación. Omitieron la respuesta un $8,5 \%$ de los encuestados.

Los porcentajes de enfermeras que decían realizar "siempre" cada tarea fueron: el $72,5 \%$ cuidados del paciente, el $78 \%$ registros asistenciales, el $50 \%$ organización de la unidad, el 36\% reunión interdisciplinaria, el

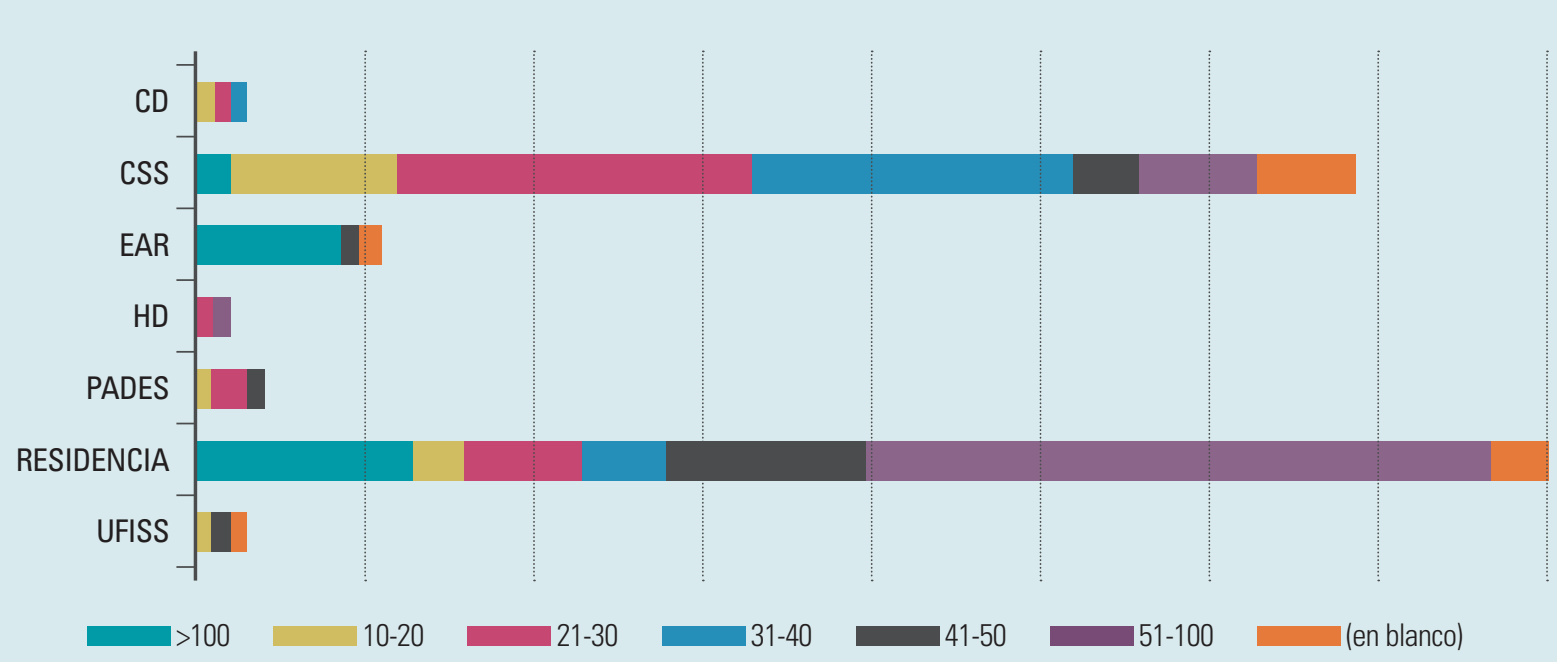

Figura 1. Ratios de pacientes por lugar de trabajo.

CD: centro de día; CSS: centro sociosanitario; EAR: equipo de atención residencial; HD: hospital de día; PADES: Programa de atención domiciliaria y equipos de soporte; UFISS: unidad funcional interdisciplinar sociosanitaria. 


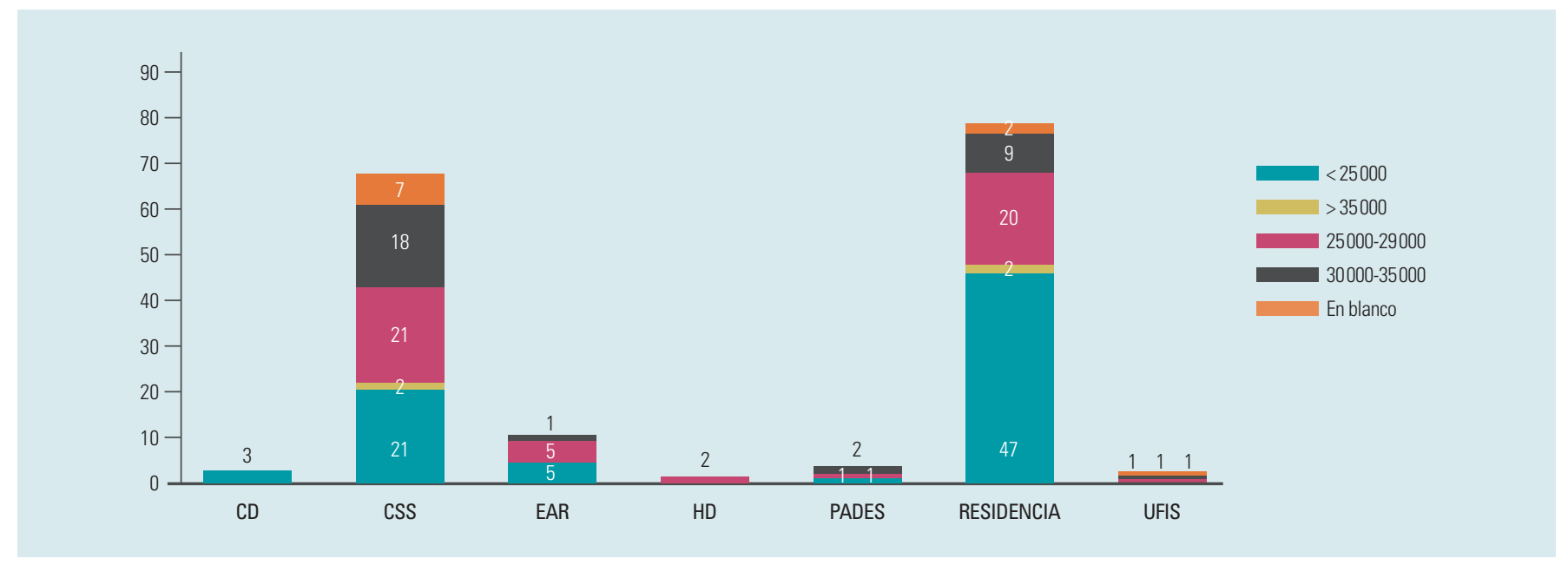

Figura 2. Sueldo con lugar de trabajo.

CD: centro de día; CSS: centro sociosanitario; EAR: equipo de atención residencial; HD: hospital de día; PADES: Programa de atención domiciliaria y equipos de soporte; UFISS: unidad funcional interdisciplinar sociosanitaria.

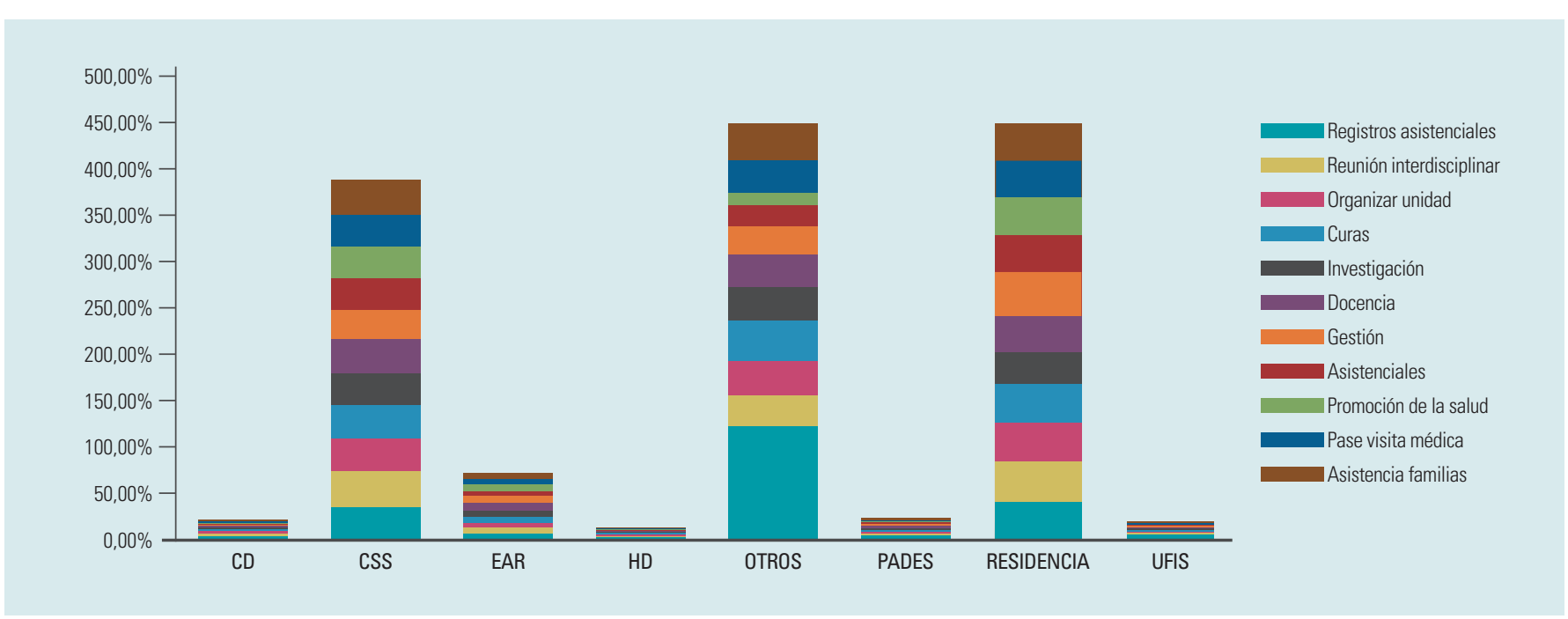

Figura 3. Tareas según recursos.

CD: centro de día; CSS: centro sociosanitario; EAR: equipo de atención residencial; HD: hospital de día; PADES: Programa de atención domiciliaria y equipos de soporte; UFISS: unidad funcional interdisciplinar sociosanitaria.

$25,5 \%$ pase de visita con el médico, el 49,5\% asistencia a familias, y omitieron la respuesta un 9,5\%. El 13\% decía dedicar menos del $25 \%$ de su jornada a la parte asistencial (omitieron la respuesta el 12\%), y solo el $6 \%$ pensaba que debería dedicar ese tiempo. El 57,5\% cree que debería dedicar más del $75 \%$ a la parte asistencial; el $12,5 \%$ omitió la respuesta.

\section{Percepción de las enfermeras del ámbito de la geriatría sobre su imagen profesional y grado de satisfacción}

En cuanto a la decisión de dedicarse a la geriatría, el 68,5\% lo hicieron por decisión propia; omitieron la respuesta un 11,5\%. Las causas de dedicarse por otros motivos fueron sobre todo porque había demanda de trabajo y era fácil encontrar más estabilidad laboral. El 69,5\% contestó que volverían a elegir dedicarse a la geriatría; omitieron la respuesta un $14,5 \%$.

El 65\% estaba satisfecho con su trabajo actual, resultado que contrastaba con que el 69\% decía sentir que no se reconoce su profesión de especialista por el resto de los profesionales de salud (omitieron la respuesta un $11,5 \%)$. Un $59 \%$ opinaba que la población no conoce la importan- cia de su trabajo y un $81 \%$ creía que no se conoce su trabajo fuera del ámbito de geriatría (omitieron la respuesta un 11\%).

\section{- DISCUSIÓN}

Las enfermeras del ámbito de la geriatría de la provincia de Barcelona son mayoritariamente mujeres con más de 15 años de profesión y con una gran experiencia en el ámbito de la geriatría. Más de la mitad tiene la especialidad en geriatría conseguida por la vía excepcional, así como también poseen formación específica en el ámbito, ya sea habiendo cursado un posgrado o un máster. Principalmente trabajan en el ámbito asistencial y están ubicadas mayoritariamente en centros sociosanitarios y residencias.

Las tres cuartas partes de las enfermeras que trabajan en geriatría tienen jornada completa; predomina especialmente las que trabajan en el turno de mańana y, además, más del $80 \%$ dispone de un contrato indefinido, lo que sugiere que estas condiciones favorecen la fidelización de las enfermeras en el ámbito de la geriatría a pesar de los sueldos. 
Tiene a su cargo un número elevado de pacientes (el 36,5\% lleva más de 50 pacientes) y evidencia que en el lugar donde existe más pacientes por enfermera es en las residencias. Hay estudios ${ }^{7-9}$, como el realizado en Suecia, que confirman este hecho y añaden que los altos grados de rotación, la insatisfacción con las condiciones, combinada con los bajos salarios y estatus, demuestran que, a más carga asistencial, más estrés y peor clima laboral ${ }^{10,11}$ de los trabajadores, lo que tiene una repercusión directa en la asistencia. En contrapartida, cabe destacar el estudio realizado por Bullich ${ }^{12}$ en centros sociosanitarios de Cataluña, en el que se puede observar que el ambiente laboral es favorable en 9 centros sociosanitarios de estudio, pero se ha de tener presente que en los centros sociosanitarios las condiciones laborales son mejores que en los centros residenciales. Como línea de investigación futura sería conveniente poder realizar un estudio comparativo entre las condiciones laborales de las residencias y los centros sociosanitarios y valorar no solo el ambiente de trabajo, sino también la satisfacción de las enfermeras.

Comparando nuestros resultados con dos estudios realizados en España ${ }^{13,14}$ se afirma que hay una relación entre los niveles de estrés y la calidad de vida profesional y que existe cansancio emocional debido a la tipología de las personas atendidas en estos recursos y a las condiciones laborales.

El recurso donde se aprecia un mejor sueldo es en el centro sociosanitario, destacando que en las residencias es donde peor se cobra. En general, la mayoría de las enfermeras encuestadas considera que el sueldo que perciben no es el adecuado.

Sorprende que más de una cuarta parte de las enfermeras entrevistadas no conocen el convenio aplicado en su lugar de trabajo.

Se observa que las tareas predominantes son asistenciales, aunque el $13 \%$ dedica menos del 25\% de la jornada a dicha labor. Cabe destacar que, al preguntar a las enfermeras por este hecho, más de la mitad opina que el tiempo dedicado a la parte asistencial debería ser mayor. Además, en la enumeración de tareas, un 58\% refiere no dedicarse nunca a tareas de investigación, porcentaje que es muy elevado. Según un estudio coreano ${ }^{15}$, las enfermeras deben proporcionar cuidados basados en la evidencia, utilizando los mejores resultados de investigación disponibles para maximizar la calidad de la atención. La utilización de la investigación es una parte importante de la práctica de enfermería basada en la evidencia y concluye que los mandos de enfermería deben facilitar el acceso a la investigación. Sin embargo, en nuestro caso, una de las causas más frecuentes de no hacer investigación es la falta de tiempo.

Sería interesante poder profundizar en cómo, cuándo, dónde y quién investiga en geriatría por parte de enfermería y los recursos que facilita la empresa para ello.

Otro hecho destacado es que más de la mitad de las encuestadas eligió el ámbito de la geriatría por elección propia y, además, ante la pregunta de si volverían a elegirlo, respondieron afirmativamente el 69,5\% de las participantes. Destacamos también que la mayoría afirma sentirse satis- fecha con su profesión, aunque no reconocida por otros profesionales, así como desconocido su trabajo por la población.

Como líneas de investigación futura creemos que hay mucho que hacer en este campo, pues no existen muchos estudios relacionados en el ámbito de la geriatría. Un estudio que consideramos interesante es valorar los factores protectores del estrés y las características de personalidad y valores que tienen las enfermeras de residencias, quienes a pesar de las condiciones laborales actuales y la carga asistencial siguen queriendo trabajar en este ámbito.

Una de las limitaciones del estudio ha sido el bajo registro de títulos de especialistas en geriatría y el bajo porcentaje de encuestas contestadas, lo que ha dificultado tener una población diana bien definida. Otra limitación es la dificultad de encontrar bibliografía relacionada directamente con el tema de estudio.

\section{- CONCLUSIONES}

La situación laboral de las enfermeras del ámbito de la geriatría de la provincia de Barcelona es de sueldos bajos, alto número de pacientes a su cargo, poco tiempo para la investigación y percepción de poco reconocimiento por parte de otros compañeros y de la población. Las enfermeras en geriatría de Barcelona sienten satisfacción profesional por su trabajo.

Las tareas predominantes son asistenciales, pero la percepción es que necesitarían dedicar más tiempo a estas tareas y menos a tareas administrativas. Cabe destacar que al preguntar a las enfermeras por este hecho más de la mitad opina que el tiempo dedicado a la parte asistencial debería ser mayor.

En cuanto a la imagen profesional y el grado de satisfacción, un alto porcentaje volvería a elegir este ámbito, aunque les gustaría tener más tiempo para la asistencia y la investigación, ya que existe la percepción de que la burocracia ocupa una gran parte de la jornada laboral.

En definitiva, tenemos unas enfermeras que realizan su trabajo por vocación. Creemos firmemente en el hecho de que si se mejoraran las condiciones laborales (sueldos, horarios y ratios) sobre todo en residencias, tendría una repercusión directa en beneficio de los pacientes. Así mejoraría la atención directa, el papel de la enfermera en geriatría dentro de la sociedad y la innovación dentro de este campo fomentando la investigación

\section{Conflicto de intereses}

Los autores declaran no tener ningún conflicto de intereses relacionado con este artículo.

\section{Agradecimentos}

Agradecemos la colaboración de todas las enfermeras que han participado en el estudio por su tiempo y dedicación, y al COIB por el soporte proporcionado.

\section{- BIBLIOGRAFÍA}

1. Martínez Cuervo F. Ya somos especialistas: ¿y ahora qué? Gerokomos. 2016; 27(2):41-4

2. Boletín Oficial del Estado, número 288 (30 de noviembre de 2009).

3. Boletín Oficial del Estado, número 213 (5 de setiembre de 2013).

4. Instituto Nacional de Estadística (INE). Provecciones de población 2016-2066. Notas de prensa [Internet]. 2016. [actualizado 20 de octubre de 2016; citado 31 de mayo de 2018]. Disponible en: https://www.ine.es/prensa/np994.pdf

5. Departamento de Salud. Generalitat de Catalunya. Plan de Salud de Cataluña 2016-2020. [Internet]. 2016. [actualizado 2016; citado
31 de mayo de 2018]. http://salutweb.gencat.cat/ca/el departament/Pla_salut/pla-de-salut-2016-2020/index.html\#googtrans\%28cales $\% 29$

6. Garcia S. Entrevista a Fernando Martínez Cuervo. La enfermería Geriátrica se ha convertido en una pieza clave en las residencias. Balance Sociosanitario de la dependencia y la discapacidad. 2014.

7. Wang Y, Dong W, Mauk K, Li P, Wan J, Yang G, et al. Nurses' Practice Environment and Their Job Satisfaction: A Study on Nurses Caring for Older Adults in Shanghai. PLoS One. 2015;10(9).
8. Petterson IL, Arnetz BB, Arnetz JE, Horte LG. Work environment, skills utilization and health of Swedish nurses - Results from a national questionnaire study. Psychother Psychosom. 1995;64(1):20-31

9. Öhman A, Keisu BI, Enberg B. Team social cohesion, professionalism, and patient-centeredness: Gendered care work, with specia reference to elderly care - a mixed methods study. BMC Health Serv Res. 2017:17(1):381

10. Sarabia-Cobo CM, Díez Saiz Z, San Millán Sierra S, Salado Morales L, Clemente Campo V. Relación entre estrés y calidad de vida en 
profesionales del área psicogeriátrica como medidor de intervención para mejora en el clima laboral. Gerokomos. 2016;27(2):48-52

11. Gómez-Cantorna C, Clemente M, Bugallo-Carrera C, Gandoy-Crego M Cuidados paliativos gerontológicos: influencia de las condiciones laborales y burnout en el personal de enfermería. Gerokomos. 2016:27(3):91-6.

12. Bullich-Marin I, Miralles Basseda R, Torres Egea P, Planas-Campmany $\mathrm{C}$, Juve-Udina ME. Evaluation of the nurse working environ- ment in health and social care intermediate care units in Catalonia Rev Esp Geriatr Gerontol. 2016;51(6):342-8.

13. Sarabia-Cobo CM, Díez Saiz Z San Millán Sierra S, Salado Morales L, Clemente Campo V. Relación entre estrés y calidad de vida en profesionales del área psicogeriátrica como medido de intervención para mejora en el clima laboral. Gerokomos. 2016;27(2):48-52.
14. Gómez-Cantorna C, Clemente M, Bugallo-Carrera C, Gandoy-Crego M. Cuidados paliativos gerontológicos: influencia de las condiciones laborales y burnout en el personal de enfermería. Gerokomos. 2016;27(3):91-6

15. Kang H. Geriatric hospital nurses' perceived barriers to research utilization and empowerment. Asian Nurs Res (Korean Soc Nurs Sci). 2015;9(1):65-72. 